\title{
Simulation on optimized complex process modeling based on XML REN Ting-yan
}

\author{
Department Of Computer Science, Qiannan Normal University Of Nationalities. Duyuan 558000 \\ China
}

Keywords: XML; multidimensional dynamic list scheduling; dynamic priority of platform

\begin{abstract}
XML complex process relationship design problem, in multidimensional dynamic list scheduling algorithm, for the defect of platform (Group) selection caused by static priority of platform, an optimized priority calculation method is designed based on XML complex process modeling, during the process of task matching, the proposed method changes dynamically for the priority of platform according to the change of task demanded resource, finally through the simulation example, verifies the superiority and applicability of the proposed method.
\end{abstract}

\section{Introduction}

The various task of future complex environment has features like complexity, distribution and changeable; at the same time, the number of resources is huge and diverse. How to process scientific configuration and deployment for available platform resources in the task process, and achieve the reasonable matching of task - platform, execute the appropriate task on the most suitable platform at the right time, right place of execution of tasks, this is "task - platform" (RT-P) design problem (i.e. task plan) in the modeling of organizational design, in essence, it is optimized problem of platform resource scheduling implementing the distributed task[1-4].

Regardless of which method is used to solve the RT-P design problem, platform selection priority have to be determined inevitably, in the research of existing "task - platform" relationship design problems, static mode are used, have plenty of problems and deficiencies.

\section{The problem description and model establishment}

First the task and platform were defined to establish the XML model:

Definition 1: Task models includes: task set $T_{s}=\left(T_{1}, T_{2}, \ldots, T_{N}\right)$ obtained by mission decomposition, wherein, $\mathrm{N}$ represents the number of tasks. Task attributes $T_{A}$ and task sequence diagram $G_{T}$, where $G_{T}$ represents the order relationship and the relationship between input and output of tasks; attribute $T_{A}$ of task $T_{i}$ includes:

1) the start time of task $S_{i}$;

2) the processing time of task $t_{i}$;

3) the resource demand vector $R_{i}=\left(r_{i 1}, r_{i 2}, \ldots r_{i L}\right)$ of task, where $\mathrm{L}$ is the number of resource types;

4) the geographical location of task $\left(x_{i}, y_{i}\right)$;

5) the complexity of tasks $T D_{i} \in\{0,1,2,3,4,5,6,7,8,9\}$;

Definition of preorder tasks have been completed, the task set ready to perform is $R E A D Y$.

Definition 2 platform is the physical carrier to provide resource capacity to execute tasks in the organization. For each platform $P_{m}(m=1,2, \ldots, K)$, K represents the number of platforms. Its major properties includes

1) the maximum moving speed of $v_{m}$ platform;

2) initial resource capability vector $R_{m}=\left(r_{m 1}, r_{m 2}, \ldots r_{m L}\right)$ of the platform, $r_{m l}(l=1,2, \ldots, L)$ refers to 
the number of resources of $l$ types obtained from the platform ${ }^{P_{m}}$;

3) platform location $\left(X_{m}, Y_{m}\right)$;

4) the basic loss rate of platform $P F_{j}$ : represents when platform $P_{j}$ executes task $T_{i}$, the loss rate of platform when the complexity is $T D_{i}=1[5]$;

Define the current collection of free platform as FREE .

Then each variable of problem is defined:

(1) the distribution variable of task - platform is $\omega_{i m}$ :

$\omega_{i m}= \begin{cases}1 & \text { the platform } P_{m} \text { is designed to } T_{i} \\ 0 & \text { otherwise }\end{cases}$

(2) Transfer variables $x_{i j m}$ of platform between tasks

$x_{i j m}=\left\{\begin{array}{cc}1 & \text { after carrying out } T_{i} \text { by platform } P_{m} \text { design to } T_{j} \\ 0 & \text { otherwise }\end{array}\right.$

And when $i=j$, the transfer variables for platform is 0 , that is to say $x_{i j m}=x_{j i m}=0(i, j=1,2, \ldots, N ; m=1,2, \ldots, K)$.

At the same time, assuming that start and termination tasks of mission as the virtual task $T_{0}$, let $x_{00 m}=0(m=1,2, \ldots, K)$.

(3) The order variables between tasks $a_{i j}$ :

$a_{i j}=\left\{\begin{array}{ccc}0 & T_{i} & \text { must be finished before } T_{j} \text { starting } \\ 1 & \text { otherwise }\end{array}\right.$

(4) the mission completion time $T$ :

$T=\max _{i=1,2, \ldots, N}\left(s_{i}+t_{i}\right)$

(5) the total redundancy degree of resources $R D$

$R D=(R M-R I) / R I$

Where the $R M$ is total resources provided by platform, its value is:

$R M=\sum_{m=1,2, \ldots, K}\left(p_{m} * \sum_{i=1}^{L} r_{m i}\right)(6)$

Where $p_{m}$ is the number of the usage of $P_{m}$ platform, $R I$ is the sum of all task resource requirements.

This paper through resource dynamic matching between the task and the platform to reduce redundancy of usage of platform resource, thus, the objective function is to minimize complete time and the value of total redundancy multiplication of resources, as:

$\min (T * R D)(7)$

Considering distributed constraints of platform, start time constraint of task and task resource requirements capacity constraints etc.:

(1) constraint distribution of platform

When $\omega_{i m}=1$, then platform $P_{m}$ must execute a task (including the starting task $T_{0}$ ) before assigned to the ${ }^{T_{i}}$, and will also be assigned to execute the next task (including termination task $T_{0}$ ), so

$$
\begin{aligned}
& \sum_{j=0}^{N} x_{j i m}-\omega_{i m}=0 \\
& (i=1,2, \ldots, N ; m=1,2, \ldots, K)
\end{aligned}
$$




$$
\begin{aligned}
& \sum_{j=0}^{N} x_{i j m}-\omega_{i m}=0 \\
& (i=1,2, \ldots, N ; m=1,2, \ldots, K)
\end{aligned}
$$

All platforms begin to execute mission at initial task, finish the mission at termination tasks i.e.:

$\sum_{j=0}^{N} x_{0 j m}=\sum_{i=0}^{N} x_{i 0 m}=1(m=1,2, \ldots, K)(10)$

(2) the task start time constraints

When $a_{i j}=0$, the task start time must satisfy the formula:

$s_{j} \geq s_{i}+t_{i}(11)$

Considering the execution relationship between task $T_{i}, T_{j}$, equation (11) is transferred into equation (12), that is:

$$
\begin{aligned}
& s_{j} \geq s_{i}+t_{i}+x_{i j m} \frac{d_{i j}}{v_{m}} \\
& (i, j=1,2, \ldots, N ; m=1,2, \ldots, K)
\end{aligned}
$$

Among them, $d_{i j}$ is the distance between $T_{i}$ and $T_{j}$, i.e.:

$$
d_{i j}=\sqrt{\left(x_{j}-x_{i}\right)^{2}+\left(y_{j}-y_{i}\right)^{2}}
$$

When $a_{i j}=1$, the task start time need to meet:

$$
s_{j} \geq s_{i}+t_{i}-T^{\prime}(14
$$

In the formula, $T^{\prime}$ is the upper bound of complete time of all task, meet $T^{\prime} \geq T$. Integrating equation (12) and (14) to get the task start time constraints:

$$
\begin{aligned}
& s_{j} \geq s_{i}+t_{i}+x_{i j m}\left(\frac{d_{i j}}{v_{m}}+a_{i j} T^{\prime}\right)-a_{i j} T^{\prime} \\
& (i, j=1,2, \ldots, N ; m=1,2, \ldots, K)
\end{aligned}
$$

(3) task resource demand constraint

The sum of resource capacity provided by platform executing task should be greater than resource requirements of the task, i.e.:

$$
\begin{aligned}
& \sum_{m=1}^{K} r_{m l} \cdot \omega_{i m} \geq r_{i l} \\
& (i=1,2, \ldots, N ; l=1,2, \ldots, L)
\end{aligned}
$$

The objective function is to minimize the multiplication of completion time of mission and resource redundancy, the model of problem is as follows:

$$
\min (T * R D)
$$




$$
\left\{\begin{array}{l}
\sum_{j=0}^{N} x_{j i m}-\omega_{i m}=0(i=1,2, \ldots, N ; m=1,2, \ldots, K) \\
\sum_{j=0}^{N} x_{i j m}-\omega_{i m}=0(i=1,2, \ldots, N ; m=1,2, \ldots, K) \\
\sum_{j=0}^{N} x_{0 j m}=\sum_{i=0}^{N} x_{i 0 m}=1 \quad(m=1,2, \ldots, K) \\
s_{j} \geq s_{i}+t_{i}+x_{i j m}\left(\frac{d_{i j}}{v_{m}}+a_{i j} T^{\prime}\right)-a_{i j} T^{\prime} \\
(i, j=1,2, \ldots, N ; m=1,2, \ldots, K) \\
\sum_{m=1}^{K} r_{m l} \cdot \omega_{i m} \geq r_{i l}(i=1,2, \ldots, N ; l=1,2, \ldots, L) \\
0 \leq T \leq T ' ; s_{i} \geq 0 \\
x_{i j m}, \omega_{i m} \in\{0,1\}
\end{array}\right.
$$

\section{Optimization of modeling process based on FCM algorithm}

This paper presents a simple, practical method to give $\mathrm{C}$ and $u_{i j}$ of FCM algorithm. The significance of FCM algorithm is to divide C individual cluster center $V=\left(V_{1}, V_{2}, \ldots, V_{c}\right)$ in the $X=\left\{X_{1}, X_{2}, \ldots, X_{n}\right\} \subset R^{n}$ collection, if the clustering center distance is far, the $X_{i}$ in same class is close, then, the division is a reasonable. To this end we use

$$
\sigma(X, V)=\frac{1}{n} \sum_{i=1}^{n} \sum_{j=1}^{c} u_{i j}^{2}\left(X_{i}-V_{j}\right)^{T}\left(X_{i}-V_{j}\right)
$$

To represent the average discrete degree of the distance between sample $X$ and cluster center $V$, use the

$$
\bar{d}(V)=\frac{1}{C_{c}^{2}} \sum_{j-1}^{c-1} \sum_{k=j-1}^{c}\left(V_{j}-V_{k}\right)^{T}\left(V_{j}-V_{k}\right)
$$

To represent the average distance of different clustering centers, classification is a reasonable.

$$
\frac{\sigma(X, V)}{\bar{d}(V)}=f(X, C)
$$

Using $f(X, C)$ can evaluate the performance of FCM algorithm. Smaller $f(X, C)$ values is better, a threshold $\varepsilon$ is given in the practical application, if $f(X, C)<\varepsilon$, then the $C$ of value small is regarded as the number of clustering. So we can calculate $C$ and $V=\left(V_{1}, V_{2}, \ldots, V_{c}\right)$ of the FCM algorithm, through the experimental data and the above methods can calculate $C$, avoid the error caused by setting $C$ manually

\section{Experiment}

On the basis of modeling, in the MATLAB platform, approximation of a nonlinear function is achieved:

$$
y=e^{-(x-1)^{2}}+e^{-(x+1)^{2}}
$$

The simulation parameters are as follows: equidistant is utilized in the interval [-3,3] to obtain 100 points, $\mathrm{m}=2,=0.001$, activation function of the hidden layer is $\log \operatorname{sig}(x)=\frac{1}{1+e^{-\lambda x}}$, the incentive function of the output layer is purelin $(\mathrm{x})=\mathrm{x}$. Figure 1 is a variation of cluster number $\mathrm{C}$, figure 2 is 
a variation of ANN error.

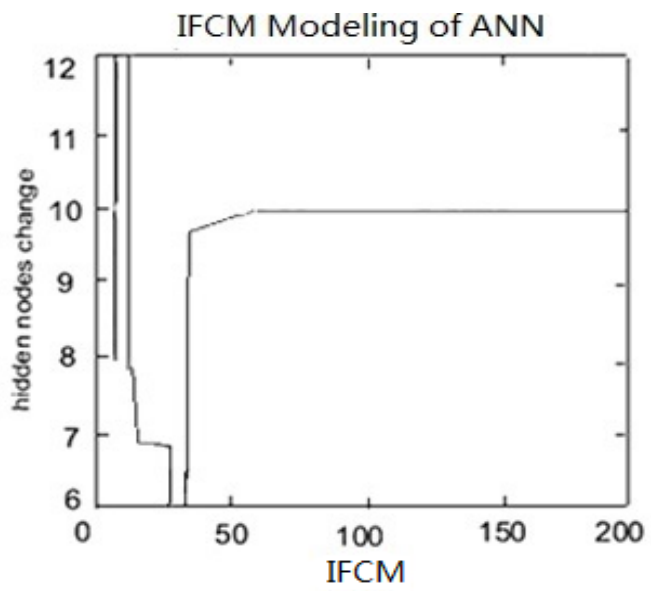

Figure 1 Variation of cluster number C

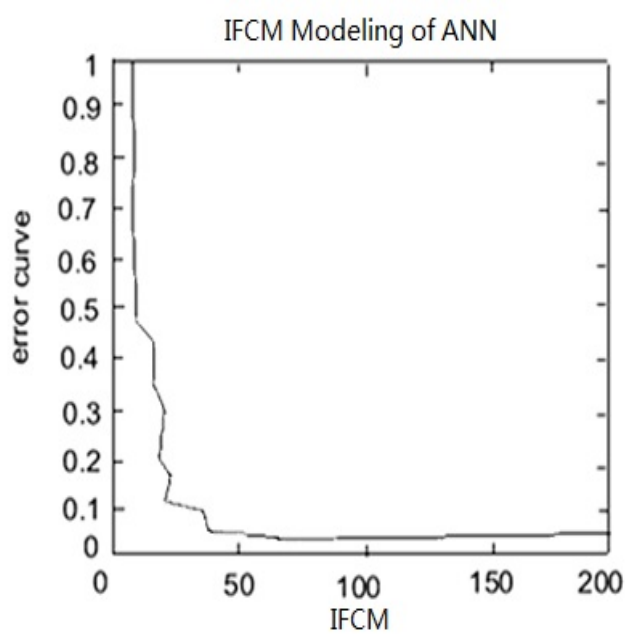

Figure 2 Error curve of ANN

The results from Figure 1 show that, the number of nodes $C$ in hidden layer is reduced from initial chaos state greatly, and stabilized at 10. From Figure 2 shows the network mean square error is decreased gradually, and "jitter" phenomenon doesn't happen. The mean square error of the network is 0.0275 , the experimental results show that, using ANN modeling based on IFCM network, can obtain the concise structure, and function approximation ability is strong, can greatly improve the mapping ability of modeling.

\section{References}

[1] Bui H, Han X, Mandal S, et al. Optimization-based Decision Support Algorithms for a Team-in-the-Loop Planning Experiment[A]. Proceedings of the 14th IEEE International Conference on Systems, Man, and Cybernetics[C]. San Antonio, TX, 2009:4684-4689.

[2] Park C, Pattipati K R, An W, et al. Quantifying the Impact of Information and Communication Structures via Distributed Auction Algorithm [A]. Proceedings of the 2010 IEEE International Conference on Systems, Man, and Cybernetics[C]. Istanbul, IEEE Press, 2010:2200-2207.

[3] Mandal S, Han X, Pattipati K R, et al. Agent-Based Distributed Framework for Collaborative Planning [A]. Proceedings of the 2010 IEEE International Conference on Aerospace conference[C]. Big Sky, MT, 2010:1-11.

[4] Liao S H. Problem structuring methods in military command and control [J]. Expert Systems with Applications, 2008, 35(3):645-653.

[5] Levchuk G M, Levchuk Y N, Luo J, et al. Normative Design of Organizations-Part I: Mission Planning [J]. IEEE Transactions on Systems, Man, and Cybernetics, 2002, 32(3): 346-359. 